\title{
Design of realistic chewing trajectory for dynamic analysis of the dental prosthesis
}

\author{
WENLONG QIN ${ }^{1}$, MING CONG $^{1}$, XIANG REN $^{2 *}$, HAIYING WEN ${ }^{3}$ \\ ${ }^{1}$ School of Mechanical Engineering, Dalian University of Technology, Dalian, China. \\ ${ }^{2}$ College of Stomatology, Dalian Medical University, Dalian, China. \\ ${ }^{3}$ School of Mechanical Engineering, Southeast University, Nanjing, China.
}

\begin{abstract}
Purpose: The chewing trajectory in the dynamic analysis of dental prosthesis is always defined as a two-segmental straight polyline without enough consideration about chewing force and motion laws. The study was aimed to design a realistic human chewing trajectory for the dynamic analysis based on force and motion planning methods. Methods: The all-ceramic crown restored in the mandibular first molar was selected as the representative prosthesis. Firstly, a dynamic model containing two molar components and one flat food component was built, and an approximate chewing plane was predefined. According to the desired forces ( $25 \mathrm{~N}, 150 \mathrm{~N}$ and $25 \mathrm{~N})$, three force planning points were calculated by using tentative trajectories. The motion planning was then executed based on four-segment cubic spline model. Finally, the new trajectory was re-imported into the dynamic model as the displacement load for evaluating its stress influence. Results: The maximum lateral velocity was $26.81 \mathrm{~mm} / \mathrm{s}$. Besides, the forces in the three force planning points were $14.11 \mathrm{~N}, 126.75 \mathrm{~N}$ and $13.56 \mathrm{~N}$. The overall repetition rate of chewing force was $77.21 \%$. The force and stress profiles were similar to the sine curve on the whole. The maximum dynamic stress of the crown prosthesis was $398.5 \mathrm{MPa}$. Conclusions: The motion law was effectively brought into the chewing trajectory to introduce the dynamic effect. The global force performance was acceptable, and the force profile was more realistic than the traditional chewing trajectory. The additional reliable characteristic feature of the stress distribution of the dental prosthesis was observed.
\end{abstract}

Key words: dental prosthesis, chewing force, chewing trajectory, dynamic analysis

\section{Introduction}

Human chewing is the predominant loading condition of the prosthesis to cause multiple failure modes [10]. The stress distribution of prosthesis during chewing in vivo is valuable data to predict prosthesis performance and improve prosthesis design. When the maximum stress during chewing is equal to the fracture strength of prosthodontic material, the loading force is regarded as the fracture strength of the prosthesis structure [9]. Fatigue life is also derived from the stress distribution during chewing, according to the structural fatigue theory [12]. Besides, the prosthesis design, including material selection and parameter determination, can be improved by comparing these different stress distributions [2], [7].

In order to obtain accurate stress distribution, finite element analysis (FEA) is requested to adopt a realistic loading condition in the occlusal phase (the effective loading time frame) as far as possible [15]. The loading condition is quite different when the test food changes. The dynamic analysis (applying displacement loading condition instead of force loading condition) is a preferable way to reflect the various chewing feature (the short occlusal time and the changing force) than the static analysis. Thus, how to design a realistic chewing trajectory for the dynamic analysis becomes a research focus [15]. It will im-

\footnotetext{
* Corresponding author: Xiang Ren, College of Stomatology, Dalian Medical University, No. 9 West Section Lvshun South Road, 116024, Dalian, China. E-mail: renxiangdy@foxmail.com

Received: February 27th, 2020

Accepted for publication: June 3rd, 2020
} 
prove the accuracy of the stress result and further help the dentist to evaluate the performance of the prosthesis. The motion and force laws learned from the clinical statistical data are two basic design principles of the chewing trajectory.

The motion law, such as the piecewise polynomial model, has been widely applied in the trajectory planning of chewing robot study [17]. The motion law is applied in the dynamic analysis in different ways. The trajectory in the concerning occlusal phase was always reduced to a two-segment straight polyline constituted by an initial intrusive point, a centric occlusal point and a final extrusive point [1]. The trajectory was also ever generated by defining the shrinkage of each biting muscle [13]. However, the trajectories did not follow the motion law as fully as possible.

The implicit feature of the chewing trajectory is generating the proper chewing force. The chewing

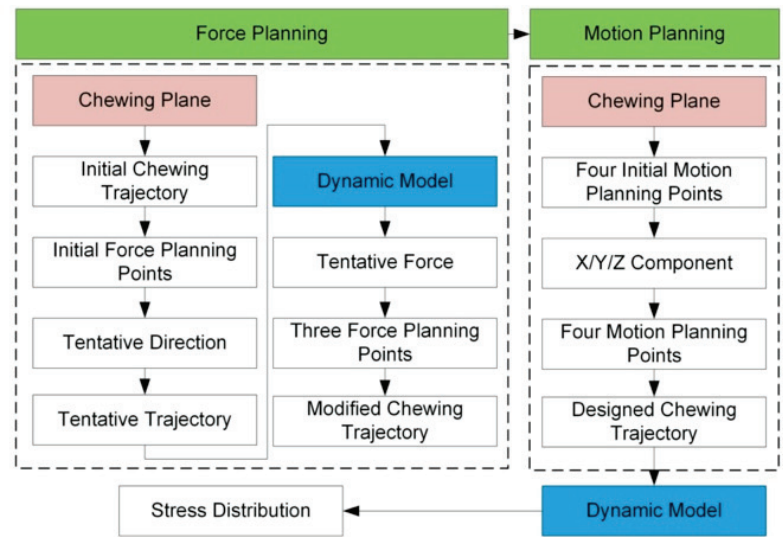

(a)
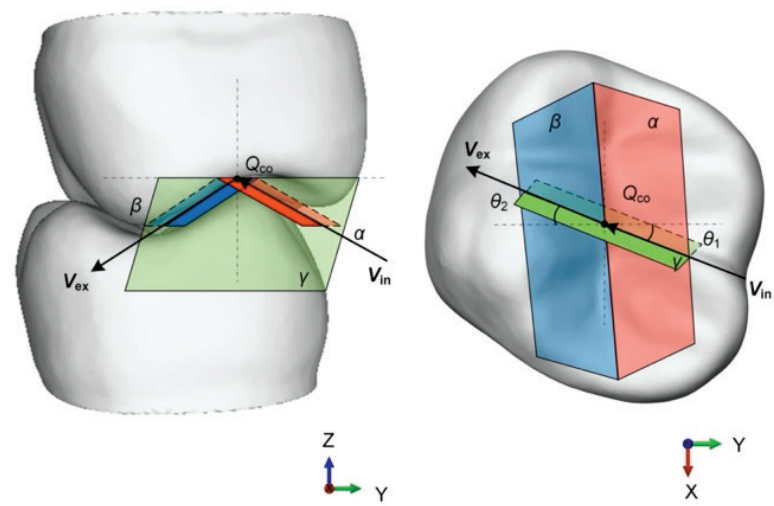

(c) force is directly loaded on the occlusal contact surface or distributed on the muscular bonding points, which is not linked to chewing trajectory [13], [14]. How to design the chewing trajectory reversely according to the determined force data is rarely researched. One attempt was adding a fixed penetration depth between upper and lower teeth on the trajectory after motion planning [8], [11]. The reasonable penetration depth could generate the desired average force during one chewing cycle (or the desired force on one typical mandibular position). However, the whole force profile (or exact forces on two or more typical mandibular positions) is still hard to realize. Besides, this method is not suitable for the dynamic model involving food.

The above traditional trajectory does not take the full motion and force achievements into consideration, making the stress distribution of the prosthesis less accurate. The study was aimed to design a more real-

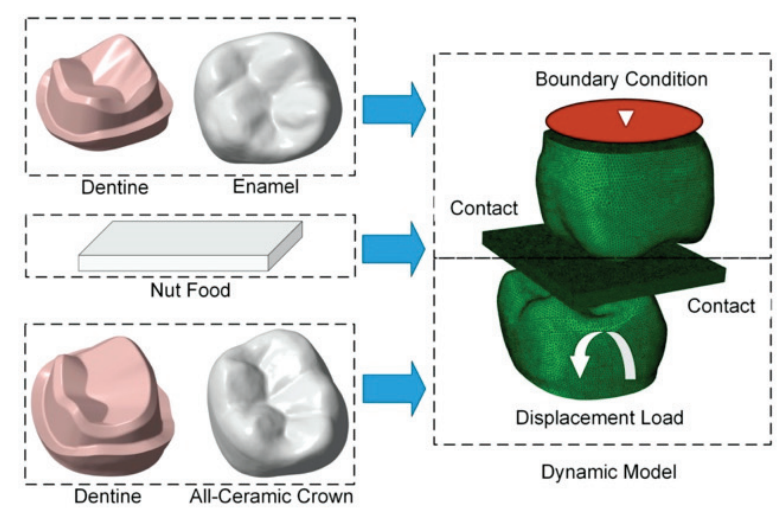

(b)

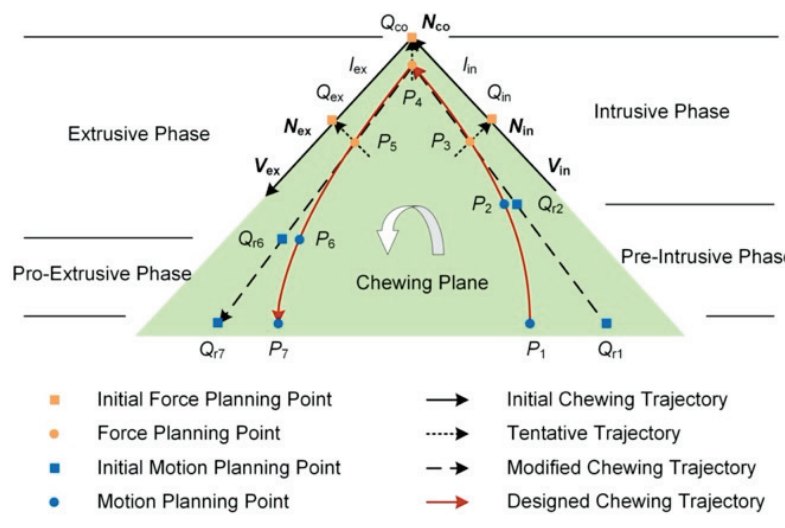

(d)

Fig. 1. Technology roadmap and explanation of critical steps (a) Technology roadmap overview; (b) Dynamic model;

(c) Chewing plane ( $\alpha$ - the lingual slopes plane of buccal cusp of the maxillary molar; $\beta$ - buccal slopes plane of lingual cusp of the maxillary molar; $\gamma-2 \mathrm{D}$ chewing plane; $\theta_{1}, \theta_{2}$ - the intrusive angle and extrusive angle in horizontal plane;

$Q_{\mathrm{co}}-$ centric occlusal point; $\boldsymbol{V}_{\mathrm{in}}$ - intersection of the plane $\gamma$ and the plane $\alpha ; \boldsymbol{V}_{\mathrm{ex}}-$ intersection of the plane $\gamma$ and the plane $\beta$ );

(d) Designed trajectory $\left(\boldsymbol{V}_{\text {in }}-\right.$ intersection of plane $\gamma$ and plane $\alpha ; \boldsymbol{V}_{\text {ex }}$ - intersection of plane $\gamma$ and plane $\beta$; $Q_{\text {in }}-$ centric intrusive point; $Q_{\mathrm{co}}$ - centric occlusal point; $Q_{\mathrm{ex}}-$ centric extrusive point, $l_{\mathrm{in}}$ - intrusive sliding displacement; $l_{\mathrm{ex}}$ - extrusive sliding displacement; $\boldsymbol{N}_{\mathrm{in}}, \boldsymbol{N}_{\mathrm{co}}$ and $\boldsymbol{N}_{\mathrm{ex}}$ - tentative directions of $Q_{\mathrm{in}}, Q_{\mathrm{co}}$ and $Q_{\mathrm{ex}} ; P_{3}, P_{4}$ and $P_{5}$ - three force planning points; $Q_{\mathrm{r} 1}, Q_{\mathrm{r} 2}, Q_{\mathrm{r} 6}$ and $Q_{\mathrm{r} 7}$ - four initial motion planning points; $P_{1}, P_{2}, P_{6}$ and $P_{7}$ - four motion planning points) 
istic chewing trajectory for the dynamic analysis of the prosthesis (with food). In this paper, the mandibular first molar with an all-ceramic crown (a nut was a simulated food) was selected as the representative prosthesis with the consideration that it is the most concerning tooth position in the dental material research. Both the motion law (polynomial model) and force law (proper force profile) were set as the planning target. A novel chewing trajectory planning algorithm was proposed for the dynamic model of that prosthesis (and the dynamic analysis was executed by the inbuilt algorithm of FEM software, which was not studied here). The technology flowchart is shown in Fig. 1a.

\section{Materials and methods}

\subsection{Dynamic model of representative prosthesis}

The teeth model consisted of two antagonistic molars and one food bolus to constitute the tooth-foodtooth contact (Fig. 1b). The maxillary first molar could be segmented into a enamel and a dentine, and the mandibular first molar could be segmented into an allceramic crown and a dentine (abutment tooth after preparation for a full porcelain crown). The food bolus was defined as an elastic rectangular flat $(13.2 \times 10$ $\times 1 \mathrm{~mm})[8]$.

The auto-mesh generating algorithm supplied by HyperWorks software (Altair Engineering, Troy, MI, USA) was adopted. The material property obtained from the previous literature was assigned to the components (Table 1) [1], [4], [8]. The following tentative trajectory or chewing trajectory was loaded on the neck plane of the mandibular molar. The fixed boundary condition was set on the neck plane of the maxillary molar. The dynamic analysis in Abaqus software (3DS, Waltham, MA, USA) was executed on the Dell Precision T7600 (Dell, Round Rock, TX, USA).

Table 1. Mechanical properties of dental and food materials

\begin{tabular}{|l|c|c|c|}
\hline \multicolumn{1}{|c|}{ Component } & $\begin{array}{c}\text { Density } \\
{\left[\mathrm{kg} / \mathrm{m}^{3}\right]}\end{array}$ & $\begin{array}{c}\text { Elastic modulus } \\
{[\mathrm{GPa}]}\end{array}$ & $\begin{array}{c}\text { Poisson's } \\
\text { ratio }\end{array}$ \\
\hline Enamel & 2958 & 84.1 & 0.30 \\
\hline Dentine & 2140 & 24.5 & 0.31 \\
\hline All-Ceramic Crown & 6100 & 220.0 & 0.30 \\
\hline Nut Food & 1000 & 0.02157 & 0.35 \\
\hline
\end{tabular}

\subsection{Constraint of chewing plane}

In order to restraint the complexity of the following force and motion planning, it was assumed that the chewing trajectory in the occlusal phase was in a $2 \mathrm{D}$ chewing plane $\gamma$ (Fig. 1c). The plane could be determined through an in-plane point and a normal vector. In detail, the in-plane point was the centric occlusal position $Q_{\mathrm{co}}$, and the normal vector of this plane was derived from the intrusive angle $\theta_{1}\left(10^{\circ}\right)$ and extrusive angle $\theta_{2}\left(10^{\circ}\right)$ in the horizontal plane.

\subsection{Force planning based on tentative behavior}

Before force planning, an initial chewing trajectory was defined as a two-segment straight polyline in the chewing plane (Fig. 1d). The centric occlusal position $Q_{\text {co }}$ was the intersection of two straight lines. The intrusive vector $\boldsymbol{V}_{\text {in }}$ was the intersection of the chewing plane $\gamma$ and the guiding plane $\alpha$ (the lingual slopes plane $\alpha$ of the buccal cusp of the maxillary molar). The extrusive vector $\boldsymbol{V}_{\mathrm{ex}}$ was the intersection of the chewing plane $\gamma$ and the plane $\beta$ (the buccal slopes plane $\beta$ of the lingual cusp of the maxillary molar). The intrusive and extrusive sliding displacements $\left(l_{\text {in }}\right.$ and $\left.l_{\text {ex }}\right)$ were $2.5 \mathrm{~mm}$ and $1.5 \mathrm{~mm}$.

A complete tentative trajectory included an origin point (an initial force planning point selected from the initial chewing trajectory, and its tentative displacement was set to $0 \mathrm{~mm}$ ), a tentative direction and a motion range (the tentative displacement was from $-3 \mathrm{~mm}$ to $0 \mathrm{~mm}$ ) (Fig. 1d). In order to make the normal contact force along the chewing trajectory reveal the desired symmetric force (similar to sine curve), three initial force planning points including the centric intrusive point $Q_{\text {in }}$ at the center of the intrusive phase, the centric occlusal point $Q_{\text {co }}$ and the centric extrusive point $Q_{\text {ex }}$ at the center of the extrusive phase were selected (Fig. 1d). The centric intrusive point and centric extrusive point were calculated by following equations. The three tentative directions were $\boldsymbol{N}_{\text {in }}$ normal to $\boldsymbol{V}_{\text {in }}$, $\boldsymbol{N}_{\text {co }}$ parallel to z-axis and $\boldsymbol{N}_{\text {ex }}$ normal to $\boldsymbol{V}_{\text {ex }}$.

$$
\begin{aligned}
& \boldsymbol{Q}_{\text {in }}=\boldsymbol{Q}_{\mathrm{co}}+0.5 \times l_{\text {in }} \times \boldsymbol{V}_{\mathrm{in}}, \\
& \boldsymbol{Q}_{\mathrm{ex}}=\boldsymbol{Q}_{\mathrm{co}}+0.5 \times l_{\mathrm{ex}} \times \boldsymbol{V}_{\mathrm{ex}} .
\end{aligned}
$$

The tentative trajectory was imported into the dynamic model as the displacement load individually. When the food was severely compressed at one tentative displacement, the dynamic analysis would termi- 
nate by itself. The normal contact force versus displacement (force range) was output. The desired chewing forces on the centric intrusive point, the centric occlusal point and the centric extrusive point were 25,150 and $25 \mathrm{~N}$, respectively [5]. The displacements, $d_{\text {in }}, d_{\mathrm{co}}$ and $d_{\mathrm{ex}}$, were planned backward according to the desired force. Then, three force planning points, $P_{3}, P_{4}$ and $P_{5}$, were calculated based on the following equations. A modified chewing trajectory represented by the two-segment straight polyline model was obtained based on the force planning method (Fig. 1d).

$$
\begin{aligned}
& \boldsymbol{P}_{3}=\boldsymbol{Q}_{\mathrm{in}}+d_{\mathrm{in}} \times \boldsymbol{N}_{\mathrm{in}}, \\
& \boldsymbol{P}_{4}=\boldsymbol{Q}_{\mathrm{co}}+d_{\mathrm{co}} \times \boldsymbol{N}_{\mathrm{co}}, \\
& \boldsymbol{P}_{5}=\boldsymbol{Q}_{\mathrm{ex}}+d_{\mathrm{ex}} \times \boldsymbol{N}_{\mathrm{ex}} .
\end{aligned}
$$

\subsection{Motion planning based on four-segment cubic spline model}

The displacement of the chewing trajectory was a 3D curve recorded by the mandibular tracking system in the previous clinical statistical literature [16]. The designed trajectory could be decomposed into three components in different directions. The trajectories in $y$ and $z$ directions were designed to be coincident with the motion law, and the trajectory in $x$-direction was directly inferred on the basis of the constraint of the chewing plane [3].

In order to realize the high-quality motion planning, four detailed chewing phases were built including pre-intrusive phase (initial moving point $P_{1}$ and initial intrusive point $P_{2}$ ), intrusive phase (initial intrusive point $P_{2}$, centric intrusive point $P_{3}$ and centric occlusal point $P_{4}$ ), extrusive phase (centric occlusal point $P_{4}$, centric extrusive point $P_{5}$ and final extrusive point $P_{6}$ ) and pro-extrusive phase (final extrusive point $P_{6}$ and final moving point $P_{7}$ ). For keeping the velocity and acceleration continuous, a four-segment cubic spline model was used to represent the trajectory in the four phases (Fig. 1d). The time point $t_{i}$ $\left(t_{1}=0 \mathrm{~s}, t_{4}=0.3 \mathrm{~s}, t_{7}=0.6 \mathrm{~s}\right.$ and the others were unknown parameters) was set on the corresponding mandibular position $P_{i}$.

Four motion planning points $\left(P_{1}, P_{2}, P_{6}\right.$ and $\left.P_{7}\right)$ were needed to be determined. A couple of equations for solving each segment cubic spline model in $z$-direction and $y$ direction are shown in Table 2. The $z$ coordinate of the four motion planning points was predefined directly. The four unknown time parameters $\left(t_{2}, t_{3}, t_{5}\right.$ and $t_{6}$ ) were adjusted dynamically to make the velocity and acceleration in $z$-direction match with the clinical record [3]. Besides, the $y$ coordinate of the four motion planning points was predefined indirectly based on the four initial motion planning points $\left(Q_{\mathrm{r} 1}\right.$, $Q_{\mathrm{r} 2}, Q_{\mathrm{r} 6}$ and $\left.Q_{\mathrm{r} 7}\right)$ from the above-modified chewing trajectory. Four uncertain shape parameters $\left(\mathrm{C}_{1}, \mathrm{C}_{2}, \mathrm{C}_{3}\right.$ and $\mathrm{C}_{4}$ ) were introduced to make the velocity and acceleration in $y$-direction identical to the clinical record [3]. A final chewing trajectory was obtained after motion planning (Fig. 1d).

The final chewing trajectory was re-imported into the dynamic model. The normal/tangential contact force along the chewing trajectory was exported to evaluate the force performance of the proposed planning method. The dynamic stress of the prosthesis on the typical planning point simultaneously output to evaluate the stress influence of the trajectory.

Table 2. Constraint equations of four-segment cubic spline models in $z$ and $y$ directions

\begin{tabular}{|l|c|c|}
\hline \multicolumn{1}{|c|}{ Phase } & $z$ Direction & $y$ Direction \\
\hline \multirow{5}{*}{ Pre-intrusive phase } & $f_{1}\left(t_{1}\right)=z_{1}$ & $g_{1}\left(t_{1}\right)=y_{\mathrm{r} 2}+C_{1}\left(y_{\mathrm{r} 1}-y_{\mathrm{r} 2}\right)$ \\
& $f_{1}\left(t_{2}\right)=f_{2}\left(t_{2}\right)$ & $g_{1}\left(t_{2}\right)=g_{2}\left(t_{2}\right)$ \\
& $f_{1}^{\prime}\left(t_{2}\right)=f_{3}^{\prime}\left(t_{1}\right)$ & $g_{1}^{\prime}\left(t_{2}\right)=g_{2}^{\prime}\left(t_{2}\right)$ \\
& $f_{1}^{\prime \prime}\left(t_{2}\right)=f_{2}^{\prime \prime}\left(t_{2}\right)$ & $g_{1}^{\prime \prime}\left(t_{2}\right)=g_{2}^{\prime \prime}\left(t_{2}\right)$ \\
\hline \multirow{5}{*}{ Intrusive phase } & $f_{2}\left(t_{4}\right)=z_{4}$ & $g_{2}\left(t_{4}\right)=y_{4}$ \\
& $f_{2}\left(t_{3}\right)=z_{3}$ & $g_{2}\left(t_{3}\right)=y_{3}$ \\
& $f_{2}\left(t_{2}\right)=z_{2}$ & $g_{2}\left(t_{2}\right)=y_{2}+C_{2}\left(y_{\mathrm{r} 2}-y_{2}\right)$ \\
& $f_{2}^{\prime}\left(t_{4}\right)=0$ & $g_{2}^{\prime}\left(t_{4}\right)=0$ \\
\hline Extrusive phase & $f_{3}\left(t_{4}\right)=z_{4}$ & $g_{3}\left(t_{4}\right)=y_{4}$ \\
& $f_{3}\left(t_{5}\right)=z_{5}$ & $g_{3}\left(t_{5}\right)=y_{5}$ \\
& $f_{3}\left(t_{6}\right)=z_{6}$ & $g_{3}\left(t_{6}\right)=y_{6}+C_{3}\left(y_{\mathrm{r} 3}-y_{6}\right)$ \\
& $f_{3}^{\prime}\left(t_{4}\right)=0$ & $g_{3}^{\prime}\left(t_{4}\right)=0$ \\
\hline Pro-extrusive phase & $f_{4}\left(t_{7}\right)=z_{7}$ & $g_{4}\left(t_{7}\right)=y_{\mathrm{r} 3}+C_{4}\left(y_{\mathrm{r} 4}-y_{\mathrm{r} 3}\right)$ \\
& $f_{4}\left(t_{6}\right)=f_{3}\left(t_{6}\right)$ & $g_{4}\left(t_{6}\right)=g_{3}\left(t_{6}\right)$ \\
& $f_{4}^{\prime}\left(t_{6}\right)=f_{3}^{\prime}\left(t_{6}\right)$ & $g_{4}^{\prime}\left(t_{6}\right)=g_{3}^{\prime}\left(t_{6}\right)$ \\
& $f_{4}^{\prime \prime}\left(t_{6}\right)=f_{3}^{\prime \prime}\left(t_{6}\right)$ & $g_{4}^{\prime \prime}\left(t_{6}\right)=g_{3}^{\prime \prime}\left(t_{6}\right)$ \\
\hline
\end{tabular}

Note:

1) $f_{i}(t)$ and $g_{i}(t)$ denote cubic spline model in $z$ and $y$ directions, respectively. When $i=1,2,3$ and 4 , the models denote preintrusive phase, intrusive phase, extrusive phase and pro-extrusive phase, respectively.

2) $t_{\mathrm{i}}$ is the time parameter of the four-segment cubic spline model. When $i=1,2,3,4,5,6$ and 7, time parameters denote initial moving time point, initial intrusive time point, centric intrusive time point, centric occlusal time point, centric extrusive time point, final extrusive time point and final moving time point. When $i=1,4$ and 7, the time parameters are $0,0.3$ and 0.6. When $i=2,3,5$ and 6 , the time parameters are unknown.

3) $y_{i}$ and $z_{i}$ denote the $\mathrm{y}$ and $\mathrm{z}$ coordinates of the motion planning points in corresponding time parameter $t_{i}$. When $i=1,2,6$ and $7, z$ coordinates are $-3.0,-1.2,0,-1.5$ and $-3.0 . y_{\mathrm{r} i}$ denotes the $\mathrm{y}$ coordinate of the initial motion planning point in corresponding time parameter $t_{\mathrm{i}}$. Four shape parameters $\left(C_{1}, C_{2}, C_{3}, C_{4}\right)$ are unknown. 


\section{Results}

The planning results of the initial chewing trajectory and the tentative trajectories are shown in Fig. 2a. The normal contact force versus displacement on the three force planning points is shown in Fig. 2b. The tentative trajectories in the three force planning points terminated at the different tentative displacements $(0,-0.32$ and $-0.13 \mathrm{~mm})$, and the forces achieved its maximum values $(44.50,187.20$ and $144.95 \mathrm{~N})$. The planned tentative displacements $(-0.10,-0.38$ and $-0.55 \mathrm{~mm}$ ) were calculated backward.

Figures $3 \mathrm{a}-\mathrm{c}$ show the motion performance of the designed chewing trajectory. The initial intrusive time point, the centric intrusive time point, the
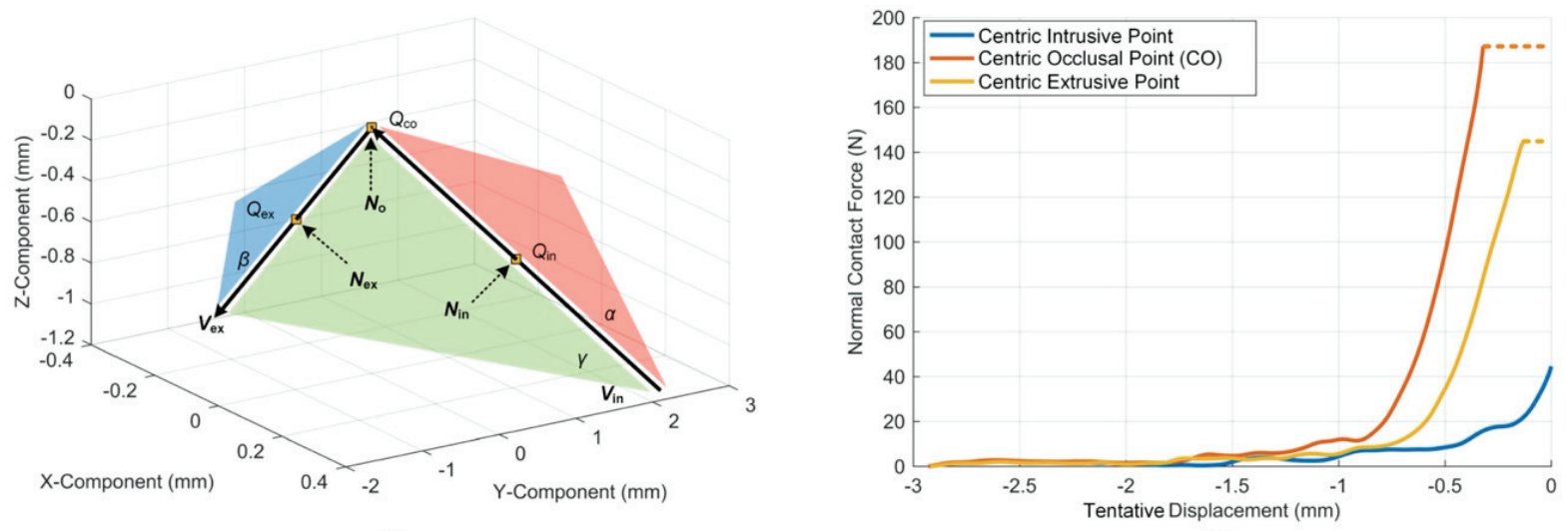

(a)

(b)

Fig. 2. Tentative trajectory and its force range: (a) Initial chewing trajectory and tentative trajectories;

(b) Normal contact force versus displacement on centric intrusive point, centric occlusal point, and centric extrusive point
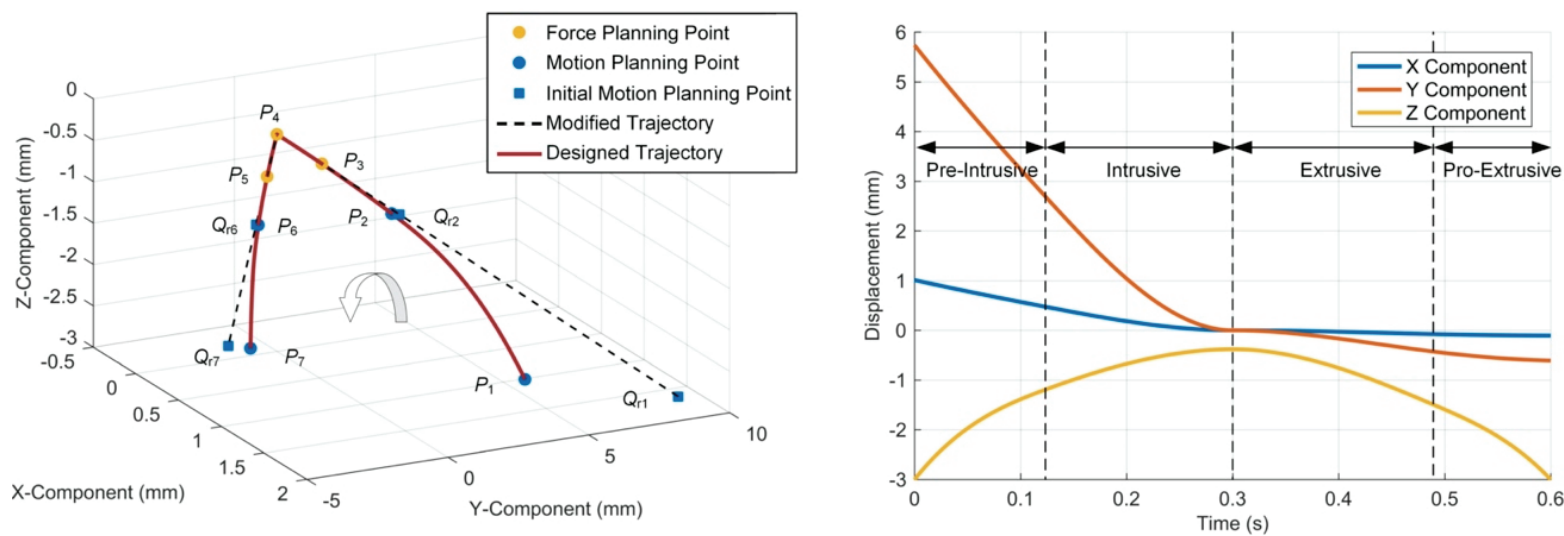

(a)

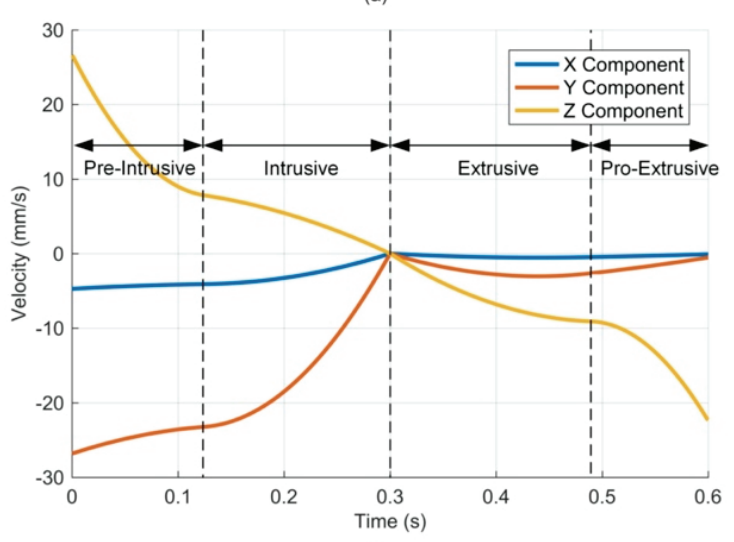

(c)

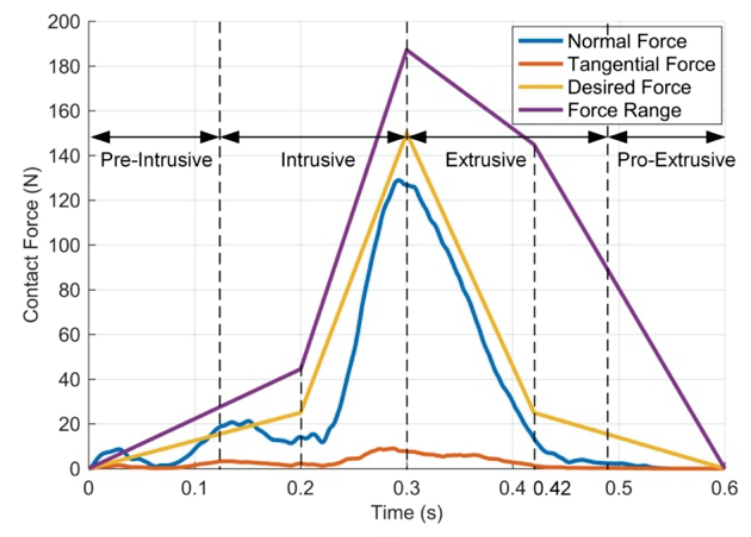

(d)

Fig. 3. Designed chewing trajectory and its force profile: (a) Chewing trajectory in 3D space.

$\left(P_{3}, P_{4}\right.$ and $P_{5}$ - three force planning points; $Q_{\mathrm{r} 1}, Q_{\mathrm{r} 2}, Q_{\mathrm{r} 6}$ and $Q_{\mathrm{r} 7}$ - four initial motion planning points;

$P_{1}, P_{2}, P_{6}$ and $P_{7}$ - four motion planning points); (b) Displacement profile; (c) Velocity profile; (d) Contact force profile 


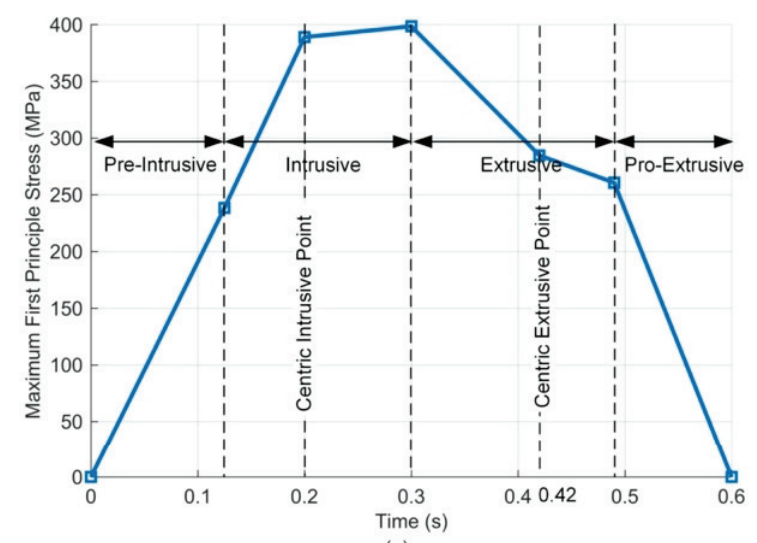

(a)

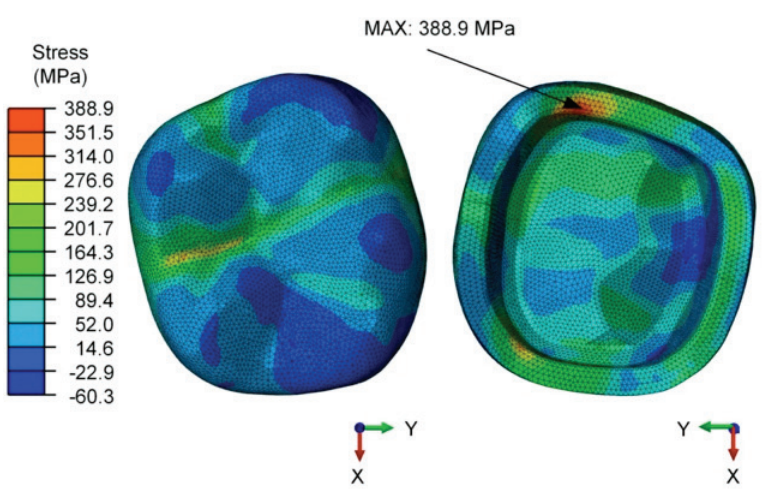

(c)

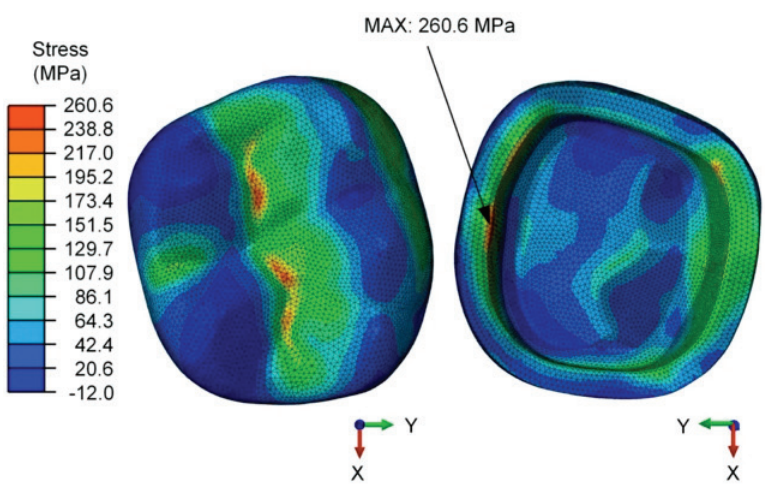

(e)

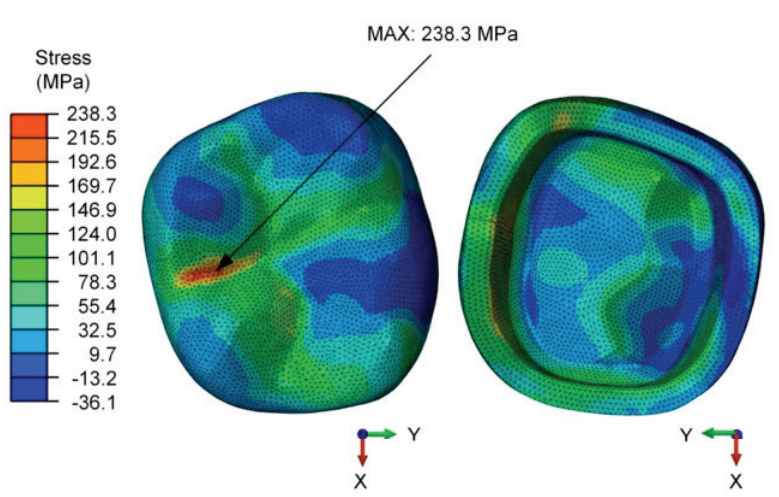

(b)

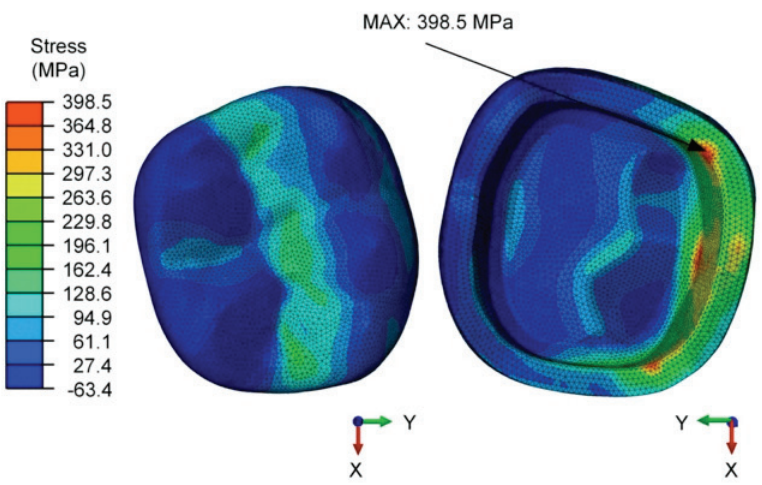

(d)

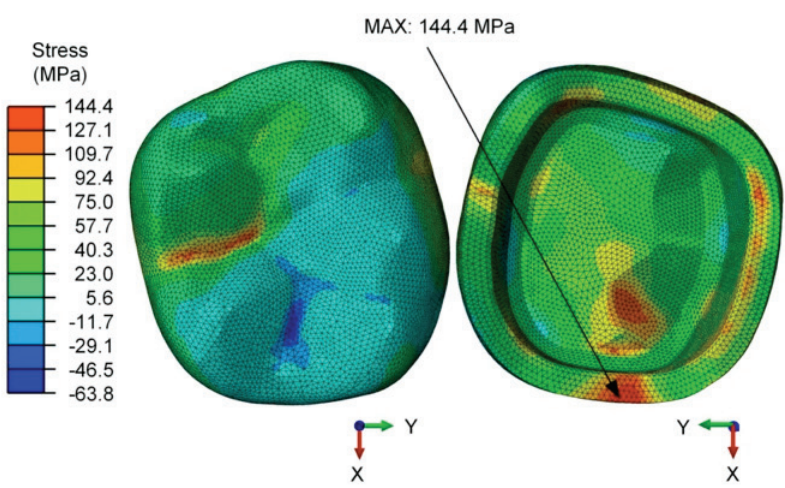

(f)

Fig. 4. Influence evaluation of designed chewing trajectory on stress distribution of crown prosthesis:

(a) Maximum first principal stress profile; (b) Stress distribution on initial intrusive point;

(c) Stress distribution on centric intrusive point; (d) Stress distribution on centric occlusal point;

(e) Stress distribution on centric extrusive point; (f) Stress distribution on final extrusive point

centric extrusive time point and final extrusive time point were adjusted to be the proper values $(0.125$, $0.2,0.42$ and $0.49 \mathrm{~s})$. The shape parameters were modified to be the proper values $(0.9,0.4,0.8$ and 0.2 ). The maximum displacements in $x, y$ and $z$ directions were $1.01,5.74$ and $3 \mathrm{~mm}$, respectively. Their velocities were $4.73,26.81$ and $26.56 \mathrm{~mm} / \mathrm{s}$. Figure $3 \mathrm{~d}$ shows the simulated normal and tangential contact forces along the designed chewing trajectory, desired force and force range. The forces on the three force planning points were 14.11, 126.75 and $13.56 \mathrm{~N}$, and the deviations were 10.89, 23.25 and $11.44 \mathrm{~N}$, respectively. The overall repetition rate was $77.21 \%$.

The stress of the prosthesis under the realistic chewing trajectory proposed here is output in Fig. 4. The maximum stresses in different chewing states were $238.3 \mathrm{MPa}, 288.9 \mathrm{MPa}, 398.5 \mathrm{MPa}, 260.6 \mathrm{MPa}$ and 144.4 MPa. The whole maximum stress appeared on the centric occlusal point. 


\section{Discussion}

\subsection{Criticism of the trajectory planning method}

In order to uncouple the force and motion request, the force planning was manually ahead of the motion planning in the paper. From an oral process point of view, the force planning method can be seen as an analogy of the "first bite" mechanism. The mechanism is that there exists a tentative trajectory in the chewing sequence as the first bite to judge the food property and feedback to the following chewing trajectory [6]. The "first bite" mechanism is a useful step for the following chewing force generation.

The tentative trajectory terminated due to the excessive element distortion of the food, which means that the force range is closely related to the food property (such as thickness and elastic modulus). Besides, the desired force value is needed to be in the force range. This constraint guarantees that the chewing trajectory after force planning will be applied successfully without the excessive element distortion. Thus, the force range indicates the possible shape of the desired force profile. If the force range covers the human maximum biting force, the chewing trajectory has the potential to represent the extreme loading condition for the dental prosthesis. Moreover, the force range can also be used to represent the chewiness of food in food texture study.

\subsection{Evaluation of the motion and force performances of the trajectory}

The desired motion law is well represented by the four-segment cubic spline model. The complete motion law is brought into the dynamic analysis. Other mathematic models, such as the eighth polynomial model, could be used to substitute the segment cubic spline model. Nevertheless, compared to the higher-order polynomial model, the lower order segmental polynomial model can avoid bad Runge's phenomenon.

The normal contact force on the force planning points in the chewing trajectory is not well coincident with the predefined planning values as desired, but the overall performance is still acceptable. When the position and deformation of the food in the tentative trajectory are the same with the one in the designed chewing trajectory, the normal contact force will be identical to the predefined force ideally. However, there still exist certain deviations due to the inevitable free movement of food. The position of the food component in the tentative trajectory is not coincident with that in the designed trajectory because of the different tangential movement. Only if the food component keeps a smaller sliding movement with the mandibular molar, the force planning method will be more precise. Although the force performance on controllable points is not well, the normal contact force profile is still realistic. It is more similar to the desired sine curve on the whole than the force profile generated by setting the fixed penetration depth (the force planning method mentioned above) [1].

\subsection{Evaluation of the trajectory effect on the stress distribution}

The stress influence of the realistic chewing trajectory proposed here was evaluated compared to other planning methods. The stress is much higher than that calculated by the single force planning method (198.80 MPa) [13]. The stress increment is caused by the dynamic effect brought from motion planning. It can be further explained by the basic kinematic equation. The additional inertia force is added into the equation. The maximum stress in the centric occlusal point is slightly larger than that calculated by the traditional chewing trajectory $(318.80 \mathrm{MPa})$ [13]. More reliable stress distribution on different points (five points here) can be output, which is also recommended to evaluate. The sine stress profile becomes available data and can be further investigated. The reliability comes from above realistic trajectory planning.

\section{Conclusions}

A realistic chewing trajectory was proposed for the dynamic analysis of dental prosthesis based on a joint planning method (force planning at first and motion planning at second). The maximum lateral velocity (y direction) was $26.81 \mathrm{~mm} / \mathrm{s}$. The motion law is effectively brought into the chewing trajectory to introduce the dynamic effect. Moreover, the forces in the three force planning points were $14.11 \mathrm{~N}, 126.75 \mathrm{~N}$ and $13.56 \mathrm{~N}$. The overall repetition rate of chewing force was $77.21 \%$. The global force performance was acceptable, although the force performance on controllable points was not well. The sine force profile was 
more realistic than the traditional chewing trajectory. Lastly, the maximum dynamic stress of the crown prosthesis was 398.5 MPa. Compared with other planning methods, the additional reliable characteristic feature of the stress distribution of the dental prosthesis is observed. The improvement of the overall force performance is to be studied in the future. This study supplies a more realistic loading condition to study other related clinical dental problems.

\section{Acknowledgements}

This work was supported by the National Natural Science Foundation of China (Grant No. 51575078 and No. 51705063).

\section{References}

[1] Benazzi S., Nguyen H.N., Kullmer O., KuPczik K., Dynamic modelling of tooth deformation using occlusal kinematics and finite element analysis, PloS One, 2016, 11(3), e152663.

[2] Bramanti E., Cervino G., Lauritano F., Fiorillo L., Amico C.D., Denaro S.S.D., Denaro S.S.D., Antonella P., MARCO C., FEM and von Mises analysis on prosthetic crowns structural elements: Evaluation of different applied materials, Sci. World J., 2017, 1-7.

[3] Buschang P.H., Hayasaki H., Throckmorton G.S., Quantification of human chewing-cycle kinematics, Arch. Oral. Biol., 2000, 45(6), 461-474.

[4] Campos T., Ramos N.C., Machado J., Bottino M.A., Souza R., MELO R.M., A new silica-infiltrated Y-TZP obtained by the sol-gel method, J. Dent., 2016, 48, 55-61.

[5] CHEN J., Food oral processing - A review, Food Hydrocolloid, 2009, 23 (1), 1-25.

[6] Dan H., Kohyama K., Interactive relationship between the mechanical properties of food and the human response during the first bite, Arch. Oral. Biol., 2007, 52 (5), 455-464.
[7] De Jager N., Pallav P., Feilzer A.J., The influence of design parameters on the FEA-determined stress distribution in CAD-CAM-produced all-ceramic dental crowns, Dent. Mater., 2005, 21 (3), 242-251.

[8] Dejak B., Mlotкowski A., 3D-Finite element analysis of molars restored with endocrowns and posts during masticatory simulation, Dent. Mater., 2013, 29 (2), 309-317.

[9] De la Rosa Castolo G., Guevara, Perez S.V., Arnoux P.J., BADIH L., BONNET F., BEHR M., Mechanical strength and fracture point of a dental implant under certification conditions: A numerical approach by finite element analysis, J. Prosthet. Dent., 2018, 119 (4), 611-619.

[10] Lodi E., Weber K.R., Benetti P., Corazza P.H., Della Bona A., BorBA M., How oral environment simulation affects ceramic failure behavior, J. Prosthet. Dent., 2018, 119 (5), 812-818.

[11] Magne P., Cheung R., Numeric simulation of occlusal interferences in molars restored with ultrathin occlusal veneers . Prosthet. Dent., 2017, 117 (1), 132-137.

[12] PÉREZ M.A., Life prediction of different commercial dental implants as influence by uncertainties in their fatigue material properties and loading conditions, Comput. Meth. Prog. Bio., 2012, 108 (3), 1277-1286.

[13] RAZAghi R., Biglari H., KARIMI A., Dynamic finite element simulation of dental prostheses during chewing using muscle equivalent force and trajectory approaches, J. Med. Eng. Technol., 2017, 41 (4), 314-324.

[14] Rezende C.E.E., Borges A.F.S., Gonzaga C.C., Duan Y., Rubo J.H., GRIGGS J.A., Effect of cement space on stress distribution in Y-TZP based crowns, Dent. Mater., 2016, 30 (12), 1304-1315.

[15] Roehrle O., Saini H., Ackland D.C., Occlusal loading during biting from an experimental and simulation point of view, Dent. Mater., 2018, 34(1), 58-68.

[16] Tanaka Y., Yamada T., Maeda Y., IKeBe K., Markerless three-dimensional tracking of masticatory movement, J. Biomech., 2016, 49 (3), 442-449.

[17] Wang G.F., Cong M., Ren X., Wen H.Y., Qin W.L., Chewing-cycle trajectory planning for a dental testing chewing robot, I. J. Robotics and Automation, 2019, 34 (3). 